\title{
A importância de Reservas Particulares do Patrimônio Natural para a conservação da brioflora da Mata Atlântica: um estudo em El Nagual, Magé, RJ, Brasil
}

\author{
Nivea Dias dos Santos ${ }^{1,3}$ e Denise Pinheiro da Costa ${ }^{2}$
}

Recebido em 12/02/2007. Aceito em 18/06/2007

\begin{abstract}
RESUMO - (A importância de Reservas Particulares do Patrimônio Natural para a conservação da brioflora da Mata Atlântica: um estudo em El Nagual, Magé, RJ, Brasil). Foi realizado o levantamento das briófitas da RPPN El Nagual, uma área de floresta submontana no Estado do Rio de Janeiro. Foram registradas 137 espécies (um antócero, 70 hepáticas e 66 musgos) e duas variedades, distribuídas em 75 gêneros e 37 famílias, sendo cinco novas ocorrências para o estado. Lejeuneaceae (27 spp.), Pilotrichaceae (17 spp.), Aneuraceae (9 spp.) e Calymperaceae (8 spp.) destacam-se pela riqueza de espécies (44\% da brioflora). Sete formas de vida foram caracterizadas, predominando trama (37\%), tufo $(16 \%)$ e talosa (15\%). Seis tipos de substrato são colonizados na área, predominando espécies corticícolas (52\%) e rupícolas (42\%). Em relação aos padrões de distribuição, predominaram espécies neotropicais e pantropicais. Foram encontradas quatro espécies caracterizadas como vulneráveis no estado. Os resultados demonstram que a brioflora da RPPN é rica e evidenciam a importância dessa categoria de unidade de conservação na proteção de remanescentes de Mata Atlântica e conservação da brioflora.
\end{abstract}

Palavras-chave: briófitas, Floresta Atlântica submontana, conservação, Rio de Janeiro

\begin{abstract}
The importance of Private Natural Heritage Reserves for conservation of Atlantic rain forest bryoflora: a study at El Nagual, Magé, Rio de Janeiro State, Brazil). A floristic survey of the bryophytes was carried out in the El Nagual Private Natural Heritage Reserve, a submontane Atlantic rain forest remnant in Rio de Janeiro state. One hundred and thirty seven species were recorded (one Anthocerotae, 70 hepatics and 66 mosses) plus two varieties, in 75 genera and 37 families. Five species are new records for Rio de Janeiro state. Lejeuneaceae (27 spp.), Pilotrichaceae (17 spp.), Aneuraceae (9 spp.), and Calymperaceae ( 8 spp.) are especially rich in species (44\% of the bryoflora). Seven life-forms were found; the most common are weft (37\%), turf (16\%), and thallose (15\%). Six kinds of substrate were colonized, the most important species types being corticicolous (52\%) and rupicolous (42\%). The most common distribution patterns were Neotropical and Pantropical. Four species were considered to be vulnerable in the state. The results show that the bryoflora of the El Nagual Reserve is relatively rich and demonstrate the importance of this conservation unit category in the protection of remnant Atlantic rain forests and in the conservation of the bryoflora.
\end{abstract}

Key words: bryophytes, submontane Atlantic rain forest, conservation, Rio de Janeiro State

\section{Introdução}

As Florestas Tropicais Úmidas abrigam provavelmente a brioflora mais rica do mundo devido à sua complexidade e variedade de microhabitats (Pócs 1982; Gradstein 1995). A Mata Atlântica, considerada um dos mais ricos conjuntos de ecossistemas do planeta (Rambaldi et al. 2003), abriga uma brioflora altamente diversa (Gradstein et al. 2001), sendo o bioma com maior riqueza de hepáticas do Brasil (Gradstein \& Costa 2003).
No Estado do Rio de Janeiro, os remanescentes florestais de Mata Atlântica representam 19\% do território (Fundação S.O.S. Mata Atlântica 2002), e embora sob pressão antrópica, ainda reservam uma considerável riqueza de briófitas, sendo reconhecidos por Costa et al. (2005a) um total de 1.036 táxons (698 musgos, 333 hepáticas e cinco antóceros).

Trabalhos clássicos, como os de Raddi (1822), Nees ab Esenbeck (1833), Hornschuch (1840), Müller (1898; 1900; 1901), Stephani (1905-1912), Dusén (1903), Brotherus (1924), Herzog (1925) entre outros,

\footnotetext{
1 Instituto de Pesquisas Jardim Botânico do Rio de Janeiro, Escola Nacional de Botânica Tropical, Rua Pacheco Leão 915, 22460-030 Rio de Janeiro, RJ, Brasil

2 Instituto de Pesquisas Jardim Botânico do Rio de Janeiro, Programa Diversidade Taxonômica, Rua Pacheco Leão 915, $22460-030$ Rio de Janeiro, RJ, Brasil

3 Autor para correspondência: nivea.dias@gmail.com
} 
foram os primeiros a tratar da brioflora do país e são essenciais para o conhecimento da diversidade de briófitas do estado. Posteriormente, inventários envolvendo a brioflora do estado foram realizados por Bartram (1954), Costa (1988; 1992; 1994; 1997; 1999), Costa \& Salamene (2003), Costa \& Yano (1988; 1995; 1998), Oliveira-e-Silva \& Yano (2000a; b), Molinaro \& Costa (2001), Costa \& Lima (2005) e Costa et al. (2006).

Costa et al. (2005b) apresentam um panorama da brioflora do estado, onde analisam a diversidade e importância das espécies de briófitas para a conservação de seus ecossistemas, indicando centros de diversidade e áreas prioritárias para levantamentos florísticos. Dentre os municípios que contribuem expressivamente para a riqueza de briófitas no Rio de Janeiro, estão Itatiaia (489 spp.), Rio de Janeiro (346 spp.), Nova Friburgo (282 spp.) e Teresópolis (272 spp.).

Apesar disso, $60 \%$ dos municípios do estado possuem sua brioflora desconhecida, e vários daqueles que apresentam registros de espécies de briófitas, tiveram suas coletas restritas a uma determinada localidade. Esse é o caso do município de Magé, para onde são conhecidas 83 espécies de briófitas, a maioria delas coletadas na Serra da Estrela, constituindo parte de coleções de naturalistas do século XIX, como Raddi e Langsdorf. A despeito dessas informações, pouco ainda é conhecido sobre a brioflora dos demais remanescentes de Mata Atlântica do município, que conta com cinco unidades de conservação em seu território (PARNA-Serra dos Órgãos, APA-Petrópolis, APA-Guapimirim, RPPN El Nagual e RPPN Querência).

Os objetivos deste estudo são contribuir para o conhecimento da brioflora em remanescentes de Mata Atlântica no estado, analisando a riqueza, composição florística, formas de vida, tipos de substrato e padrões de distribuição das espécies de briófitas ocorrentes na Reserva Particular do Patrimônio Natural (RPPN) El Nagual; e subsidiar o plano de manejo da RPPN com dados da brioflora.

\section{Material e métodos}

Área de estudo - A RPPN El Nagual, criada em 1999, localiza-se no distrito de Santo Aleixo, município de

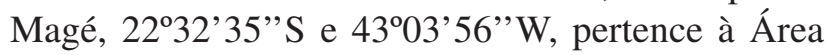
de Proteção Ambiental de Petrópolis, fazendo parte da Serra dos Órgãos, com uma área de 17,20 ha e altitude variando entre 170-450 m. Apresenta trechos característicos de forte declividade com formação de talvegues. A pluviosidade varia entre 1.000 e $2.200 \mathrm{~mm}$ e o clima da área pode ser caracterizado como quente e úmido, com temperatura média anual de $20^{\circ} \mathrm{C}$ (Ministério do Meio Ambiente \& O Instituto Ambiental, 2005 inédito).

A formação ocorrente em El Nagual está enquadrada na fitofisionomia Floresta Ombrófila Densa Submontana (Veloso et al. 1991). Em quase sua totalidade, a área é coberta por formações florestais de mata secundária em estágios médio ou avançado de regeneração, dependendo da altitude considerada. Nas cotas mais elevadas da reserva, existem remanescentes de formações primárias (Ministério do Meio Ambiente \& O Instituto Ambiental 2005, inédito).

Amostragem e estudo do material - Entre fevereiro e agosto/2005 foram realizadas cinco excursões para coleta de briófitas na RPPN El Nagual. As coletas foram realizadas aleatoriamente nos diferentes ambientes encontrados: floresta submontana, capoeira, mata de galeria do Rio das Pedras Negras e córregos do interior da floresta. As metodologias de coleta, herborização e preservação do material seguem Yano (1984a).

A identificação foi realizada principalmente com base na seguinte literatura: Buck (1998), Costa (2007), Crum \& Anderson (1981), Dauphin (2003), Frahm (1991), Gradstein \& Costa (2003), Gradstein et al. (2001), Hedenäs (2003), Heinrichs et al. (1988; 2000), Hell (1969), Ochi (1980; 1981), Oliveira-e-Silva \& Yano (2000a; b), Peralta (dados não publicados), Reese (1993), Reiner-Drehwald (2000), Reiner-Drehwald \& Goda (2000), Sharp et al. (1994) e Vaz-Imbassahy \& Costa (2006a; b).

A classificação adotada é a proposta por Stotler \& Crandall-Stotler (2005) para a Divisão Anthocerotophyta, Crandall-Stotler \& Stotler (2000) para a Divisão Marchantiophyta e Buck \& Goffinet (2000) para a Divisão Bryophyta.

Todos os exemplares coletados estão depositados no herbário do Jardim Botânico do Rio de Janeiro (RB), com duplicatas no herbário da Faculdade de Formação de Professores da Universidade do Estado do Rio de Janeiro (HFFP).

Análise dos dados - Para cada táxon foram analisadas as seguintes características: forma de vida, tipo de substrato, variação altitudinal no Brasil, e distribuição geográfica no Brasil e no mundo. Também foi realizada uma análise da riqueza e composição florística da brioflora da RPPN. Para as formas de vida foi adotada 
a classificação de Mägdefrau (1982), com modificações feitas por Richards (1984). Em relação à forma de vida pendente, foram reconhecidos dois tipos: pendente (eixo principal pendente com base aderida ao substrato e eixos secundários curtos e horizontais) e flabelado (eixos com ramos num mesmo plano, projetados horizontal a obliquamente para baixo, com filídios aplanados).

A nomenclatura adotada para os tipos de substrato segue a de Robbins (1952), sendo consideradas corticícolas, espécies que crescem sobre troncos; epífilas, aquelas que crescem sobre folhas; epíxilas, sobre troncos em decomposição; rupícolas, sobre rocha; e terrícolas, sobre solo.

Os dados de variação altitudinal no Brasil e distribuição geográfica no país e no mundo foram baseados nos trabalhos de Gradstein \& Costa (2003), Yano $(1981 ; 1984 b ; 1989 ; 1995 ; 2006)$, complementados com informações do banco de dados da brioflora do Estado do Rio de Janeiro.

Na distribuição dos táxons no Brasil, a seqüência dos estados é apresentada por região geográfica e os nomes estão abreviados de acordo com o IBGE, como apresentado a seguir: Região Norte (RR - Roraima, RO - Rondônia, AP - Amapá, AC - Acre, AM - Amazonas, PA - Pará, TO - Tocantins); Região Nordeste (MA - Maranhão, PI - Piauí, CE - Ceará, RN - Rio Grande do Norte, PB - Paraíba, PE - Pernambuco, AL - Alagoas, SE - Sergipe, BA - Bahia); Região Centro-oeste (GO - Goiás, MT- Mato Grosso, MS - Mato Grosso do Sul); Região Sudeste (MG - Minas Gerais, ES - Espírito Santo, RJ - Rio de Janeiro, SP - São Paulo); Região Sul (PR - Paraná, SC - Santa Catarina, RS - Rio Grande do Sul).

\section{Resultados e discussão}

Riqueza - Foram coletadas 268 amostras, sendo duas de antóceros, 121 de hepáticas e 145 de musgos, totalizando 137 espécies de briófitas (um antócero, 70 hepáticas e 66 musgos) e duas variedades, distribuídas em 75 gêneros e 38 famílias (Tab. 1), o que representa $13 \%$ das espécies, $24 \%$ dos gêneros e $40 \%$ das famílias conhecidas para o Estado do Rio de Janeiro (Costa et al. 2005a). Comparando os resultados obtidos com outros inventários realizados em remanescentes de Mata Atlântica do Estado (Costa 1992; 1994; 1997; 1999; Costa \& Salamene 2003; Oliveira-e-Silva \& Yano 2000a; b), nota-se que a RPPN El Nagual, com uma área de 17 ha de floresta submontana, apresenta uma brioflora rica e representativa dos remanescentes de Mata Atlântica do Estado (13\%). Estes resultados são inferiores somente aos obtidos por Oliveira-e-Silva \& Yano (2000a; b), que encontraram 231 espécies de briófitas (22\%), para uma área no litoral sul do Estado, com 10.700 ha e diferentes formações de Mata Atlântica (terras baixas, submontana e montana). Essa riqueza, possivelmente, está relacionada ao fato da RPPN fazer parte da Serra dos Órgãos, pertencendo à zona de amortecimento do PARNA-Serra dos Órgãos; e pelo bom estado de conservação desse remanescente florestal, com diferentes ambientes (córregos, rios, florestas e capoeiras), diversidade de microhabitats para o desenvolvimento das briófitas (barrancos, rochas, troncos, etc.), e fontes de manutenção da umidade (neblina noturna, nascentes, córregos e rios não poluídos).

Os dados aqui apresentados corroboram as observações de Richards (1954) e Pócs (1982), quanto a diversidade de briófitas nas florestas tropicais estar diretamente relacionada à variedade de microhabitats para o seu desenvolvimento, a diversidade de microclimas e variedade de substratos disponíveis para plantas pequenas. Esses resultados demonstram a importância das RPPNs para a conservação da brioflora da Mata Atlântica no Estado.

Composição florística - Em termos de gêneros e espécies, a família Lejeuneaceae é a mais representativa na RPPN El Nagual, com 19 gêneros e 27 espécies (Tab. 1), correspondente a $20 \%$ da brioflora, o que era esperado, visto que esta é a maior família de hepáticas dos trópicos (Gradstein et al. 2001). $\mathrm{Na}$ RPPN El Nagual foram encontrados $45 \%$ dos gêneros e $23 \%$ das espécies de Lejeuneaceae ocorrentes no Rio de Janeiro (Costa et al. 2005a). Dentre as 27 espécies da família, três são novas ocorrências para o Estado: Diplasiolejeunea cavifolia, Drepanolejeunea biocellata e Taxilejeunea obtusangula.

Em relação à riqueza específica, também se destacam as famílias Pilotrichaceae com 17 espécies (12\%), Aneuraceae com nove espécies (6\%), Calymperaceae com oito espécies (6\%), Geocalycaceae, Neckeraceae e Plagiochilaceae com seis espécies cada (4\%). Esse resultado também era esperado, visto que essas famílias estão entre as principais em inventários brioflorísticos no Neotrópico, sendo comuns nas florestas submontanas (Gradstein \& Pócs 1989; Gradstein et al. 2001; Costa \& Lima 2005). 
Tabela 1. Lista das espécies de briófitas da RPPN El Nagual, Magé, RJ, Brasil, com dados de forma de vida (FV), tipo de substrato (TS), variação altitudinal no Brasil, distribuição no mundo e no Brasil e Voucher. Forma de vida: $\mathrm{CX}=$ coxim, $\mathrm{DN}=$ dendróide, $\mathrm{P}_{1}=$ pendente, $\mathrm{P}_{2}=$ flabelado, $\mathrm{TF}=$ tufo, $\mathrm{TL}=$ talosa, $\mathrm{TP}=$ tapete, $\mathrm{TR}=$ trama; tipo de substrato: $\mathrm{A}=$ artificial, $\mathrm{C}=$ corticícola, $\mathrm{EF}=$ epífila, $\mathrm{EX}=$ epíxila, $\mathrm{R}=$ rupícola, $\mathrm{T}=$ terrícola; voucher: $\mathrm{S}=$ Santos, $\mathrm{S} \& \mathrm{C}=\mathrm{Santos} \&$ Costa, $\mathrm{S} \& \mathrm{~V}=\mathrm{Santos} \& \mathrm{Vaz}-\mathrm{Imbassahy}$ * * ocorrência nova para o estado do Rio de Janeiro. Entre parênteses, ao lado de cada divisão e família, estão indicados os números de famílias, gêneros e espécies.

\begin{tabular}{|c|c|c|c|c|c|}
\hline Espécies & FV & TS A & Altitude $\mathrm{Br}(\mathrm{m})$ & Distribuição geográfica no mundo e no Brasil & Voucher \\
\hline \multicolumn{6}{|l|}{ ANTHOCEROTOPHYTA (1/1/1) } \\
\hline \multicolumn{6}{|l|}{ DENDROCEROTACEAE (1/1) } \\
\hline * Megaceros vincentianus (Lehm. \& Lindenb.) Campb. & TL & $\mathrm{R}$ & $500-1.500$ & Neotropical; PE e SP & S \& C 338 \\
\hline \multicolumn{6}{|l|}{ MARCHANTYOPHYTA $(13 / 35 / 70)$} \\
\hline \multicolumn{6}{|l|}{ ANEURACEAE (2/9) } \\
\hline Aneura pinguis (L.) Dumort. & TL & $\mathrm{R}$ & $0-800$ & Ampla; MS, MG, RJ e SP & S \& C 216 \\
\hline Riccardia amazonica (Spruce) S.W. Arnell & $\mathrm{TL}$ & $\mathrm{R}$ & $0-2.200$ & Afro-Americana; AP, AM, PA, PE, BA, GO, MG, ES, RJ e SP & S \& C 262 \\
\hline R. digitiloba (Spruce ex Steph. ) Págan & TL & EX & $0-1.400$ & Neotropical; AC, AM, CE, PE, MT, MS, MG, ES, RJ, e SP & S \& C 346 \\
\hline R. cataractatum (Spruce) Schiffn. & TL & $\mathrm{R}$ & $0-1.800$ & Paraguai, Brasil e Bolívia; MT, MS, RJ, SP e SC & S \& C 371 \\
\hline R. chamedryfolia (With.) Grolle & TL & $\mathrm{T}$ & $250-1.800$ & Regiões temperadas do hemisfério norte e Brasil; GO, RJ, SP e ES & S \& C 164 \\
\hline R. emarginata (Steph.) Hell & $\mathrm{TL}$ & $\mathrm{R}$ & $0-1.000$ & Brasil; MG, RJ e SP & S \& C 167 \\
\hline R. fucoidea (Sw.) Schiffn. & $\mathrm{TL}$ & $\mathrm{R}$ & $0-1.500$ & Neotropical; BA, RJ e SP & S \& C 362 \\
\hline R. metzgeriiformmis (Steph.) R.M. Schust. & $\mathrm{TL}$ & $\mathrm{T}$ & $0-800$ & América do Sul Tropical; AP, RJ e SP & $\mathrm{S} \& \mathrm{C} 350$ p.p. \\
\hline R. regnellii (Aongstr.) Hell & $\mathrm{TL}$ & $\mathrm{R}$ & $0-1.000$ & Brasil; PE, MG, RJ, SP, SC e RS & S \& C 106 \\
\hline \multicolumn{6}{|l|}{ BRYOPTERIDACEAE $(1 / 1)$} \\
\hline Bryopteris filicina $(\mathrm{Sw}$.$) Nees$ & $\mathrm{P}_{1}$ & $\mathrm{C}$ & $0-2.000$ & $\begin{array}{l}\text { Neotropical; RR, AM, PA, CE, PE, GO, MT, MS, MG, ES, RJ, } \\
\text { SP, PR, SC e RS }\end{array}$ & S \& C 327 \\
\hline \multicolumn{6}{|l|}{ CEPHALOZIELLACEAE $(1 / 1)$} \\
\hline Cylindrocolea sp. & $\mathrm{TP}$ & EX & - & - & S \& C 310 \\
\hline \multicolumn{6}{|l|}{ GEOCALYCACEAE (2/6) } \\
\hline Heteroscyphus combinatus (Nees) Schiffn. & TR & $\mathrm{R}$ & $0-1.200$ & Neotropical; AM, MG, RJ e SP & $\mathrm{S} \& \mathrm{C} 85$ p.p. \\
\hline Lophocolea bidentata (L.) Dumort. & TR & EX, T & $0-1.500$ & Ampla; RR, AC, AM, CE, GO, MT, MS, MG, ES, RJ, SP, PR e RS. & S \& C 219 \\
\hline L. mandonii Steph. & TR & $\mathrm{R}, \mathrm{T}$ & $1.000-2.700$ & Bolívia e Brasil; MG e RJ & S \& C 351 \\
\hline L. martiana Nees & TR & $\mathrm{EX}, \mathrm{R}, \mathrm{T}$ & $0-1.850$ & $\begin{array}{l}\text { Afro-americana; AP, AM, PA, CE, PE, SE, BA, MT, MG, ES, RJ, } \\
\text { SP, PR, SC e RS }\end{array}$ & S \& C 180 \\
\hline L. muricata (Lehm.) Nees & $\mathrm{TR}$ & $\mathrm{C}$ & $250-2.300$ & $\begin{array}{l}\text { Regiões temperadas do Hemisfério Sul e montanhas tropicais; } \\
\text { MG, ES, RJ, SP e RS }\end{array}$ & S s.n. \\
\hline L. perissodonta (Spruce) Steph. & $\mathrm{TR}$ & $\mathrm{R}$ & $0-1.500$ & Neotropical; AP, AM, PA, MG, RJ, SP e SC & S \& C 203 \\
\hline \multicolumn{6}{|l|}{ JUBULACEAE $(1 / 4)$} \\
\hline Frullania arecae (Spreng.) Gottsche & $\mathrm{TP}$ & $\mathrm{C}$ & $0-2.000$ & Pantropical; GO, MT, ES, RJ, SP, PR e RS & \\
\hline F. caulisequa (Nees) Nees & $\mathrm{TP}$ & $\mathrm{C}, \mathrm{EF}, \mathrm{EX}$ & $0-1.000$ & Neotropical; RR, AC, PA, CE, PE, SE, BA, GO, MG, ES, RJ, SP, SC e RS & S \& C 192 \\
\hline F. kunzei (Lehm. \& Lindenb.) Lehm. \& Lindenb. & $\mathrm{TP}$ & $\mathrm{C}$ & $0-2.400$ & Neotropical; AC, AM, PA, CE, PB, PE, SE, BA, MT, MG, ES, RJ, SP, PR, RS & S \& C 272 \\
\hline F. riojaneirensis (Raddi) Aongstr. & $\mathrm{TP}$ & $\mathrm{C}, \mathrm{R}$ & $0-1.100$ & Pantropical; PA, CE, PB, PE, SE, BA, GO, MT, MS, MG, ES, RJ, SP, PR e RS & S S \& C 271 \\
\hline \multicolumn{6}{|l|}{ LEJEUNEACEAE $(18 / 26)$} \\
\hline Aphanolejeunea paucifolia (Spruce) E. Reiner & TR & $\mathrm{EF}$ & $0-1.350$ & Argentina, Paraguai e Brasil; MG, ES, RJ e SP & $\mathrm{S} \& \mathrm{C} 245$ p.p. \\
\hline Archilejeunea parviflora (Nees) Schiffn. & TR & $\mathrm{C}, \mathrm{EX}$ & $0-1.500$ & Neotropical; RR, RO, AC, AM, PA, PE, MS, MG, ES, RJ e SP & S \& C 266 p.p. \\
\hline Ceratolejeunea cornuta (Lindenb.) Schiffn. & $\mathrm{TR}$ & $\mathrm{C}, \mathrm{EF}, \mathrm{R}$ & $0-1.000$ & Neotropical; RR, RO, AP, AC, AM, PA, CE, PE, MG, RJ, SP, PR e SC & $\begin{array}{l}\mathrm{S} \& \mathrm{C} 237 \\
\text { continuc }\end{array}$ \\
\hline
\end{tabular}




\begin{tabular}{|c|c|c|c|c|c|}
\hline Espécies & FV & TS & Altitude $\mathrm{Br}(\mathrm{m})$ & Distribuição geográfica no mundo e no Brasil & Voucher \\
\hline C. cubensis (Mont.) Schiffn. & TR & $\mathrm{C}, \mathrm{EF}$ & $0-500$ & Neotropical; RO, AC, AM, PA, CE, PB, PE, BA, ES, RJ e SP & S \& C 191 \\
\hline Cheilolejeunea holostipa (Spruce) R.-L. Zhu \& Grolle & $\mathrm{TR}$ & $\mathrm{C}$ & $0-2.200$ & Neotropical; PA, PE, BA, MG, ES, RJ e PR & S \& C 286 \\
\hline C. rigidula (Mont.) R.M. Schust. & TR & $\mathrm{R}$ & $0-1.000$ & $\begin{array}{l}\text { Afro-americana; RR, AC, AP, AM, PA, CE, SE, AL, PB, PE, BA, GO, MT, } \\
\text { MS, MG, ES, RJ, SP, PR e SC }\end{array}$ & S \& C 275 \\
\hline Colojeneunea obliqua (Nees \& Mont. ) Schiffn. & $\mathrm{TP}$ & $\mathrm{EF}$ & $0-300$ & Neotropical; AM, PA, PE, BA, RJ, SP, PR e SC & $\mathrm{S} \& \mathrm{C} 220$ p.p. \\
\hline C. subcardiocarpa Tixier & TR & $\mathrm{EF}$ & $0-2.000$ & Neotropical; AM, PE, MG, ES, RJ, SP, PR e SC & S \& C 289 p.p. \\
\hline Colura tenuicornis (A. Evans) Steph. & $\mathrm{TR}$ & $\mathrm{EF}$ & $0-2.000$ & Pantropical; PE, MG, ES, RJ, SP, SC e RS & S \& C 244 \\
\hline Diplasiolejeunea alata Jovet-Ast & TP & $\mathrm{EF}$ & $700-1.500$ & Neotropical; RJ, SP e PR & S \& C 285 \\
\hline * D. cavifolia Steph & $\mathrm{TP}$ & $\mathrm{EF}$ & $0-800$ & Pantropical; CE, PE, SP, PR e SC & S \& C 195 \\
\hline * Drepanolejeunea biocellata A. Evans & TP & $\mathrm{EF}$ & $0-900$ & Neotropical; SP & S \& C 187 \\
\hline D. mosenii (Steph.) Bischl. & $\mathrm{TP}$ & $\mathrm{C}, \mathrm{EF}$ & $0-2.000$ & Neotropical; AM, PE, BA, MG, ES, RJ, SP, PR, SC e RS & S \& C 285 p.p. \\
\hline Harpalejeunea oxyphylla (Nees \& Mont.) Steph. & TR & $\mathrm{C}$ & $0-1.000$ & Neotropical; AM, PA, PE, PB, BA, RJ e SP & S \& C 320 p.p. \\
\hline Lejeunea cf. cristulata (Steph.) E. Reiner \& Goda & TR & $\mathrm{R}$ & $900-1.500$ & Brasil; PE, MG, RJ, SP e SC & S \& C 198 p.p. \\
\hline L. flava (Sw.) Nees & TR & $\mathrm{C}, \mathrm{EF}$ & $0-2.400$ & $\begin{array}{l}\text { Pantropical; RR, AC, AM, PA, CE, PE, GO, BA, MT, MS, MG, ES, } \\
\text { RJ, SP e RS }\end{array}$ & S \& C 321 \\
\hline L. laetevirens Nees \& Mont. & TR & $\mathrm{C}$ & $0-1.500$ & $\begin{array}{l}\text { Neotropical; RR, AC, AM, PA, CE, PB, PE, AL, BA, GO, MT, MS, } \\
\text { ES, RJ e SP }\end{array}$ & S \& C 303 \\
\hline Leptolejeunea elliptica (Lehm. \& Lindenb.) Schiffn. & $\mathrm{TP}$ & $\mathrm{EF}$ & $0-1.500$ & $\begin{array}{l}\text { Neotropical; RR, AP, AC, AM, PA, CE, PE, AL, BA, GO, MT, MG, ES, } \\
\text { RJ, SP, PR e SC }\end{array}$ & S \& C 289 \\
\hline $\begin{array}{l}\text { Leucolejeunea xanthocarpa (Lehm. \& Lindenb.) } \\
\text { A. Evans }\end{array}$ & TR & $\mathrm{C}$ & $0-2.500$ & Pantropical; CE, PE, BA, MG, ES, RJ, SP, SC e RS & S \& C 272 p.p. \\
\hline Lopholejeunea subfusca (Nees) Schiffn. & TR & $\mathrm{C}$ & $0-750$ & $\begin{array}{l}\text { Pantropical; RR, RO, AC, AM, PA, CE, PB, PE, BA, GO, MT, MS, } \\
\text { MG, ES, RJ, SP e SC. }\end{array}$ & S \& C 153 \\
\hline Marchesinia brachiata (Sw.) Schiffn. & TR & $\mathrm{C}$ & $0-1.500$ & $\begin{array}{l}\text { Neotrópico e Leste da África; RR, CE, SE, PE, BA, MT, MG, ES, RJ, } \\
\text { PR e SC }\end{array}$ & $\mathrm{S} \& \mathrm{C} 365$ p.p. \\
\hline Microlejeunea bullata (Taylor) Steph. & TR & $\mathrm{C}$ & $0-2.400$ & Neotropical; RR, AC, AM, CE, PE, SE, BA, MT, MS, ES, RJ e SP & $\mathrm{S} \& \mathrm{C} 347$ p.p. \\
\hline Stictolejeunea squamata (Willd.) Schiffn. & TR & $\mathrm{C}$ & $0-1.500$ & Neotropical; AC, AM, PA, PE, BA, MG, RJ e SP & $\mathrm{S} \& \mathrm{C} 259$ p.p. \\
\hline Symbiezidium barbiflorum (Lindenb. \& Gottsche) Steph. & TR & $\mathrm{C}$ & $0-1.500$ & Neotropical; AM, PA, PE, BA, ES, RJ, SP e SC & S \& C 288 \\
\hline Taxilejeunea lusoria (Lindenb. \& Gottsche) Steph. & TR & $\mathrm{C}$ & $0-800$ & Neotropical; AM, PA, RJ, SP, PA e SC & S \& C 303 \\
\hline * T. obtusangula (Spruce) A. Evans & TR & $\mathrm{C}$ & $0-1.050$ & Neotropical; RR, PA, SP e PR & S \& C 188 \\
\hline \multicolumn{6}{|l|}{ LEPIDOZIACEAE $(2 / 2)$} \\
\hline Arachniopsis diacantha (Mont.) M.A. Howe & TR & $\mathrm{R}, \mathrm{T}$ & $0-1.000$ & Pantropical; AC, AM, PA, PE, GO, ES, RJ, SP, PR e RS & S \& C 270 \\
\hline Bazzania longistipula (Lindenb.) Trevis & TR & $\mathrm{C}$ & $400-2.400$ & Neotropical; MG, RJ, SP e PR & S \& C 83 \\
\hline \multicolumn{6}{|l|}{ MARCHANTIACEAE $(2 / 2)$} \\
\hline Dumortiera hirsuta (Sw.) Nees & $\mathrm{TL}$ & $\mathrm{R}$ & $0-2.000$ & Ampla; AC, AM, PA, MT, GO, MS, MG, ES, RJ, SP, PR, SC e RS & S \& C 215 \\
\hline Marchantia chenopoda $\mathrm{L}$. & TL & $\mathrm{R}$ & $0-1.500$ & Neotropical; AM, MT, GO, MG, ES, RJ, SP, PR e RS & S \& C 214 \\
\hline \multicolumn{6}{|l|}{ METZGERIACEAE (1/5) } \\
\hline Metzgeria albinea var. albinea Spruce & $\mathrm{TL}$ & $\mathrm{C}$ & $0-1.800$ & Pantropical; CE, PE, BA, GO, MG, ES, RJ, SP, PR, SC e RS & S \& C 387 \\
\hline M. albinea var. angusta (Steph.) Costa \& Gradst. & $\mathrm{TL}$ & $\mathrm{C}$ & $0-1.800$ & América tropical e subtropical; RJ, SP, PR e RS & $\mathrm{S} \& \mathrm{C} 365$ p.p. \\
\hline M. aurantiaca Steph. & $\mathrm{TL}$ & $\mathrm{C}$ & $0-1.600$ & Neotropical; AM, PB, PE, BA, MG, ES, RJ, SP, PR, SC e RS & $\begin{array}{l}\mathrm{S} \& \mathrm{C} 322 \\
\text { continua }\end{array}$ \\
\hline
\end{tabular}




\begin{tabular}{|c|c|c|c|c|c|}
\hline Espécies & FV & TS & Altitude $\mathrm{Br}(\mathrm{m})$ & Distribuição geográfica no mundo e no Brasil & Voucher \\
\hline M. ciliata Raddi & $\mathrm{TL}$ & $\mathrm{C}$ & $0-2.500$ & Pantropical; PE, PB, MG, ES, RJ, SP, SC e RS & S \& C 197 \\
\hline M. lechleri Steph. & $\mathrm{TL}$ & $\mathrm{C}$ & $0-2.500$ & Neotropical; CE, PE, GO, MG, ES, RJ, SP, PR, SC e RS & S \& C 273 \\
\hline M. myriopoda Lindenb. & $\mathrm{TL}$ & $\mathrm{C}$ & $0-2.000$ & Neotrópico e EUA; PE, GO, MG, ES, RJ, SP, SC e RS & S \& C 230 \\
\hline \multicolumn{6}{|l|}{ MONOCLEACEAE (1/1) } \\
\hline Monoclea gottschei Lindenb. & $\mathrm{TL}$ & $\mathrm{R}$ & $0-2.000$ & América tropical e subtropical; AM, CE, PE, RJ, SP e RS & S \& C 385 \\
\hline \multicolumn{6}{|l|}{ PALLAVICINIACEAE (2/3) } \\
\hline Pallavicinia lyellii (Hook.) S.F. Gray & TL & $\mathrm{R}$ & $100-1.000$ & Ampla; AM, PA, BA, GO, MS, RJ, SP, SC e RS & S \& C 348 \\
\hline Symphyogyna aspera Steph. & $\mathrm{TL}$ & $\mathrm{R}$ & $0-2.200$ & Neotropical; AM, PA, CE, PE, BA, MT, MS, MG, ES, RJ, SP, SC e RS & S \& C 212 \\
\hline S. brasiliensis (Nees) Nees \& Mont. & $\mathrm{TL}$ & $\mathrm{T}$ & $0-1.850$ & Afro-americana; RR, RO, CE, BA, GO, MT, MG, ES, RJ, SP, PR, SC e RS & S \& C 176 \\
\hline \multicolumn{6}{|l|}{ PLAGIOCHILACEAE $(1 / 6)$} \\
\hline Plagiochila corrugata (Nees) Nees \& Mont. & $\mathrm{P}_{1}$ & $\mathrm{C}$ & $0-2.300$ & Neotropical; PE, BA, GO, MG, ES, RJ, SP, PR, SC e RS & S \& C 347 \\
\hline P. martiana (Nees) Lindenb. & $\mathrm{P}_{1}^{1}$ & $\mathrm{C}, \mathrm{EX}, \mathrm{R}$ & $0-1.100$ & Neotropical; AC, PE, GO, MS, MG, RJ, SP, SC e RS & S \& C 229 \\
\hline P. patentissima Lindenb. & $\mathrm{P}_{1}^{1}$ & $\mathrm{R}$ & $0-1.400$ & Neotropical; PE, MG, ES, RJ, SP, PR, SC e RS & S \& C 198 \\
\hline P. patula (Sw.) Lindenb. & $\mathrm{P}_{1}^{1}$ & $\mathrm{C}, \mathrm{R}$ & $0-900$ & Neotropical; AC, BA, MG, RJ e SP & S \& C 232 \\
\hline P. raddiana Lindenb. & $\mathrm{P}_{1}^{1}$ & $\mathrm{C}$ & $0-1.350$ & Neotropical; AC, AM, PA,CE, PE, MT, MG, ES, RJ, SP, PR, SC e RS & S \& C 365 \\
\hline P. subplana Lindenb. & $\mathrm{P}_{1}^{1}$ & $\mathrm{C}, \mathrm{R}$ & $0-1.300$ & Neotropical; AM, PA, BA, MG, RJ, SP e SC & S \& C 253 \\
\hline \multicolumn{6}{|l|}{ RADULACEAE (1/4) } \\
\hline Radula javanica Gottsche & $\mathrm{P}_{2}$ & $\mathrm{C}$ & $0-1.000$ & Pantropical; AP, AM, PA, PE, BA, MG, RJ, SP e RS & S \& C 254 \\
\hline R. ligula Steph. & $\mathrm{TP}^{2}$ & $\mathrm{R}$ & $0-800$ & Brasil e Norte da Argentina; RJ, PR, SC e RS & S \& C 256 \\
\hline R. nudicaulis Steph. & $\mathrm{P}_{2}$ & $\mathrm{C}, \mathrm{R}, \mathrm{T}$ & $800-2.700$ & América do Sul Tropical e Macronésia (Madeira); MG, ES, RJ e SP & S \& C 177 \\
\hline R. recubans Tayl. & $\mathrm{P}_{2}^{2}$ & $\mathrm{C}$ & $800-1.000$ & América tropical e subtropical; PA, RJ, SP e RS & S \& C 77 \\
\hline \multicolumn{6}{|l|}{ BRYOPHYTA $(24 / 39 / 66)$} \\
\hline \multicolumn{6}{|l|}{ AMBLYSTEGIACEAE $(1 / 1)$} \\
\hline *Amblystegium cf. serpens (Hedw.) BSG & TR & $\mathrm{C}$ & - & Ampla. & S \& C 109 \\
\hline \multicolumn{6}{|l|}{ BARTRAMIACEAE $(1 / 1)$} \\
\hline Philonotis uncinata (Schwägr.) Brid. & $\mathrm{TF}$ & $A, R$ & $0-1.350$ & $\begin{array}{l}\text { Ampla; AP, AC, AM, PA, TO, PI, PB, PE, BA, GO, MT, MS, MG, ES, } \\
\text { RJ, SP e SC }\end{array}$ & S \& C 388 \\
\hline \multicolumn{6}{|l|}{ BRACHYTECIACEAE (1/1) } \\
\hline Meteoridium remotifolium (Müll. Hal.) Manuel & $\mathrm{P}_{1}$ & $\mathrm{C}, \mathrm{EX}$ & $0-2.200$ & $\begin{array}{l}\text { Neotropical; RR, AP, AC, PB, PE, BA, GO, MT, MG, ES, RJ, SP, } \\
\text { PR, SC e RS }\end{array}$ & S \& C 236 \\
\hline \multicolumn{6}{|l|}{ BRYACEAE (1/1) } \\
\hline Bryum densifolium Brid. & TF & $\mathrm{R}, \mathrm{T}$ & $0-1.200$ & Neotrópico e Austrália; RR, AP, TO, MT, BA, MG, RJ, SP, PR, SC e RS & S \& C 181 \\
\hline \multicolumn{6}{|l|}{ CALYMPERACEAE (3/8) } \\
\hline Calymperes lonchophyllum Schwägr. & $\mathrm{TF}$ & $\mathrm{EX}, \mathrm{R}$ & $0-1.200$ & Pantropical; RO, AC, TO, PE, AL, BA, GO, MT, MS, MG, ES, RJ, SP e PR & S \& C 205 \\
\hline C. palisotii Schwägr. & $\mathrm{TF}$ & $\mathrm{C}, \mathrm{R}$ & $0-500$ & $\begin{array}{l}\text { Ampla; RR, RO, AP, AM, PA, PI, RN, PB, PE, AL, SE, BA, GO, MT, } \\
\text { MG, ES, RJ, SP e PR }\end{array}$ & S \& C 202 \\
\hline C. tenerum Müll. Hal. & TF & EX & $0-200$ & Pantropical; TO, MG e RJ & S \& C 301 \\
\hline Octoblepharum albidum Hedw. & $\mathrm{TF}$ & $\mathrm{C}$ & $0-1.200$ & $\begin{array}{l}\text { Pantropical (alcançando o SE dos EUA); RR, RO, AP, AC, AM, PA, TO, } \\
\text { MA, PI, CE, RN, PB, PE, AL, SE, BA, GO, MT, MS, MG, ES, RJ, SP, } \\
\text { PR, SC e RS }\end{array}$ & $S \& C 241$ \\
\hline
\end{tabular}




\begin{tabular}{|c|c|c|c|c|c|}
\hline Espécies & $\mathrm{FV}$ & TS & Altitude $\mathrm{Br}(\mathrm{m})$ & Distribuição geográfica no mundo e no Brasil & Voucher \\
\hline O. pulvinatum (Dozy \& Molk.) Mitt. & $\mathrm{TF}$ & $\mathrm{C}, \mathrm{R}$ & $0-2.000$ & $\begin{array}{l}\text { Neotropical; RR, RO, AP, AC, AM, PA, TO, MA, CE, PE, BA, GO, MT, } \\
\text { MS, MG, ES, RJ, SP e SC }\end{array}$ & S \& C 355 \\
\hline Syrrhopodon guadichaudii Mont. & $\mathrm{TF}$ & EX & $0-1.200$ & $\begin{array}{l}\text { Pantropical; RR, AP, AM, PA, PE, BA, GO, MT, MS, MG, ES, RJ, } \\
\text { SP, PR, SC e RS }\end{array}$ & S \& C 344 \\
\hline S. incompletus Schwagr. & $\mathrm{TF}$ & $\mathrm{C}$ & $0-800$ & $\begin{array}{l}\text { Afro-americana; RR, RO, AP, AC, AM, PA, TO, PE, BA, GO, MT, MS, } \\
\text { MG, RJ, SP, PR e SC. }\end{array}$ & $\mathrm{S} \& \mathrm{C} 235$ \\
\hline S. ligulatus Mont. & $\mathrm{TF}$ & EX & $0-1.000$ & $\begin{array}{l}\text { Neotrópico e EUA; RR, RO, AP, AC, AM, PA, PE, BA, MT, MS, } \\
\text { MG, RJ e SP }\end{array}$ & S \& C 339 \\
\hline \multicolumn{6}{|l|}{ DALTONIACEAE (1/1) } \\
\hline \multicolumn{5}{|l|}{ DICRANACEAE (4/5) } & $S \& C 255$ \\
\hline Bryohumbertia filifolia var. humilis (Mont.) J.-P. Frahm & $\mathrm{TF}$ & $\mathrm{R}$ & $100-2.500$ & Ampla; AM, BA, PE, MG, RJ, SP, PR, SC e RS & S \& C 277 \\
\hline Campylopus cryptopodioides Broth. & $\mathrm{TF}$ & $\mathrm{R}$ & $0-1.500$ & Brasil e Argentina; AP, GO, MS, MG, RJ, SP, PR e RS & S \& C 298 \\
\hline C. occultus Mitt. & TF & $\mathrm{R}$ & $0-2.500$ & América do Sul; RR, AP, BA, GO MT, MS, MG, ES, RJ, SP, PR, SC e RS & S \& C 280 \\
\hline Dicranella hilariana (Mont.) Mitt. & $\mathrm{TF}$ & $\mathrm{T}$ & $0-2.000$ & $\begin{array}{l}\text { Neotropical; RR, RO, AP, AC, AM, PA, TO, CE, PB, PE, BA, GO, MT, } \\
\text { MS, MG, ES, RJ, SP e SC }\end{array}$ & S \& C 111 \\
\hline $\begin{array}{l}\text { Leucoloma serrulatum Brid. } \\
\text { FISSIDENTACEAE }(1 / 4)\end{array}$ & \multicolumn{4}{|c|}{ FISSIDENTACEAE (1/4) } & S \& C 170 \\
\hline Fissidens asplenioides Hedw. & $\mathrm{TF}$ & $\mathrm{R}$ & $0-2.200$ & Pantropical; AP, AC, MT, MG, RJ, SP, SC, PR e RS & S \& C 239 \\
\hline F. flaccidus (Mitt.) Mitt. & $\mathrm{TF}$ & $\mathrm{T}$ & $0-1.100$ & $\begin{array}{l}\text { Afro-americana; RO, AP, AC, PA, TO, CE, PB, PE, BA, GO, MT, MS, } \\
\text { MG, RJ, SP e PR }\end{array}$ & S \& C 120 \\
\hline F. prionodes Mont. & $\mathrm{TF}$ & $\mathrm{R}, \mathrm{T}$ & $0-1.500$ & $\begin{array}{l}\text { Neotropical; RR, RO, AC, AM, PA, TO, PI, PB, PE, BA, GO, MT, MG, } \\
\text { RJ, SP, PR e SC }\end{array}$ & $S \& C 251$ \\
\hline F. submarginatus Bruch & TF & $\mathrm{C}$ & $0-500$ & $\begin{array}{l}\text { Neotrópico, Sul da África e Havaí; RR, RO, AC, AM, PA, TO, PI, CE, } \\
\text { PE, BA, GO, MT, MS, MG, RJ, SP e SC }\end{array}$ & S \& C 359 \\
\hline \multicolumn{6}{|l|}{ HYPNACEAE (2/3) } \\
\hline Chryso-hypnum diminutivum (Hampe) W.R. Buck & $\mathrm{TP}$ & $\mathrm{C}$ & $0-1.200$ & $\begin{array}{l}\text { Ampla; RO, AP, AC, AM, PA, TO, PE, BA, GO, MT, MS, MG, ES, RJ, } \\
\text { SP, PR, SC e RS }\end{array}$ & $\mathrm{S} \& \mathrm{C} 315$ \\
\hline C. elegantulum (Hook.) Hampe & $\mathrm{TP}$ & $\mathrm{R}$ & $0-1.200$ & Neotropical; RO, AP, AC, AM, PE, GO, MT, MS, MG, RJ, SP, PR e SC. & S \& C 269 \\
\hline Vesicularia vesiculares var. vesicularis (Schwägr.) Broth. & $\mathrm{TP}$ & $\mathrm{R}, \mathrm{T}$ & $0-800$ & $\begin{array}{l}\text { Neotropical (alcançando o sudeste dos EUA); RO, AP, AC, AM, PA, TO, } \\
\text { PI, PE, BA, GO, MT, MS, MG, ES, RJ, SP, PR, SC e RS }\end{array}$ & S \& C 178 \\
\hline $\begin{array}{l}\text { V. vesiculares var. portoricensis (Brid.) W.R. Buck } \\
\text { HYPOPTERIGYACEAE (1/1) }\end{array}$ & $\mathrm{TP}$ & $\mathrm{R}$ & $0-1.100$ & Neotropical; AP, AM, PE, BA, GO, MS, MG, RJ, SP, PR e SC & S \& C 361 \\
\hline $\begin{array}{l}\text { Hypopterygium tamarisci (Sw.) Brid. } \\
\text { LEMBOPHYLLACEAE }(2 / 2)\end{array}$ & $\mathrm{DN}$ & $\mathrm{R}$ & $0-1.350$ & Neotropical; BA, RJ, SP, PR, SC e RS & S \& C 152 \\
\hline Orthostichella pentasticha (Brid.) W.R. Buck & $\mathrm{P}_{1}$ & $\mathrm{C}$ & $0-1.100$ & Afro-americana; AM, PE, MG, RJ e SP & S \& C 157 \\
\hline $\begin{array}{l}\text { Pilotrichella flexilis (Hedw.) Aongstr. } \\
\text { LEUCOBRYACEAE (1/1) }\end{array}$ & $\mathrm{P}_{1}$ & $\mathrm{C}$ & $0-2.300$ & Neotropical; AP, BA, MT, MS, RJ, SP, MG, PR, SC e RS & S \& C 250 \\
\hline Leucobryum martianum (Hornsch.) Hampe & $\mathrm{CX}$ & $\mathrm{R}, \mathrm{T}$ & $0-1.000$ & $\begin{array}{l}\text { Neotropical; RR, RO, AP, AM, AC, PA, TO, MA, CE, PE, AL, SE, BA, } \\
\text { GO, MT, MS, MG, ES, RJ, SP, PR, SC e RS }\end{array}$ & S \& C 279 \\
\hline
\end{tabular}

Leskeodon aristatus (Geh \& Hampe) Broth.

Bryohumbertia filifolia var. humilis (Mont.) J.-P. Frahm

Campylopus cryptopodioides Broth.

C. occultus Mitt.

Leucoloma serrulatum Brid.

NTACEAE (1/4)

issidens asplenioides Hedw.

Frionodes Mont.

F. submarginatus Bruch

YPNACEAE $(2 / 3)$

Chryso-hypnum diminutivum (Hampe) W.R. Buck 


\begin{tabular}{|c|c|c|c|c|c|}
\hline Espécies & $\mathrm{FV}$ & TS & Altitude $\mathrm{Br}(\mathrm{m})$ & Distribuição geográfica no mundo e no Brasil & Voucher \\
\hline Leucobryum martianum (Hornsch.) Hampe & $\mathrm{CX}$ & $\mathrm{R}, \mathrm{T}$ & $0-1.000$ & $\begin{array}{l}\text { Neotropical; RR, RO, AP, AM, AC, PA, TO, MA, CE, PE, AL, SE, BA, } \\
\text { GO, MT, MS, MG, ES, RJ, SP, PR, SC e RS }\end{array}$ & S \& C 279 \\
\hline \multicolumn{6}{|l|}{ METEORIACEAE (2/2) } \\
\hline Floribundaria flaccida (Mitt.) Broth. & $\mathrm{P}_{1}$ & $\mathrm{C}$ & $0-1.200$ & Neotropical; AP, BA, MT, MS, MG, RJ, SP, PR, SC e RS & S \& C 227 \\
\hline Zelometeorium patulum (Hedw.) Manuel & $P_{1}^{1}$ & $\mathrm{C}$ & $0-1.400$ & $\begin{array}{l}\text { Neotropical; RR, RO, AP, AC, AM, PA, TO, CE, PE, AL, BA, GO, MT, } \\
\text { MS, MG, ES, RJ, SP, PR, SC e RS }\end{array}$ & S \& C 225 \\
\hline \multicolumn{6}{|l|}{ MNIACEAE (1/1) } \\
\hline $\begin{array}{l}\text { Plagiomnium rhynchophorum (Hook.) T.J. Kop. } \\
\text { NECKERACEAE }(2 / 6)\end{array}$ & \multicolumn{4}{|c|}{ NECKERACEAE $(2 / 6)$} & S \& C 122 \\
\hline Neckeropsis disticha (Hedw.) Kindb. & $P_{1}$ & $\mathrm{C}, \mathrm{R}$ & $0-1.100$ & $\begin{array}{l}\text { Pantropical (alcançando o sudeste dos EUA); RR, RO, AC, AM, TO, PE, } \\
\text { BA, GO, MT, MS, MG, ES, RJ, SP, PR, SC e RS }\end{array}$ & S \& C 193 \\
\hline N. undulata (Hedw.) Reichardt & $\mathrm{P}_{1}$ & $\mathrm{R}$ & $0-1.200$ & $\begin{array}{l}\text { Américas; RR, RO, AP, AC, AM, PA, TO, MA, CE, PE, AL, BA, GO, MT, } \\
\text { MS, MG, RJ, SP, PR, SC e RS }\end{array}$ & S \& C 306 \\
\hline Porotrichum longirostre (Hook.) Mitt. & $\mathrm{DN}$ & $\mathrm{C}$ & $800-2.700$ & Neotropical; MT, MS, RJ, SP, SC e RS & S \& C 252 \\
\hline P. korthalsianum (Dozy \& Molk.) Mitt. & $\mathrm{DN}$ & $\mathrm{R}$ & $200-2.000$ & Neotropical; BA, RJ, SP e RS & S \& C 221 \\
\hline P. mutabile Hampe & $\mathrm{DN}$ & $\mathrm{C}$ & $0-1.700$ & Neotropical; BA, RJ, SC e RS & S \& C 323 \\
\hline P. substriatum (Hampe) Mitt. & $\mathrm{DN}$ & $\mathrm{C}$ & $0-2.000$ & Afro-americana; RO, AC, TO, PE, BA, GO, RJ, SP, SC e RS & S \& C 259 \\
\hline \multicolumn{6}{|l|}{ PHYLLOGONIACEAE $(1 / 1)$} \\
\hline Phyllogonium viride Brid. & $\mathrm{P}_{1}$ & $\mathrm{C}$ & $0-2.300$ & Afro-americana; AP, CE, PE, AL, BA, MT, MG, ES, RJ, SP, PR, SC e RS & S \& C 144 \\
\hline \multicolumn{6}{|l|}{ PILOTRICHACEAE (7/17) } \\
\hline Callicostella apophysata (Hampe) A. Jaeger & $\mathrm{TR}$ & $\mathrm{EX}, \mathrm{R}$ & $0-400$ & Brasil; GO e RJ & S \& C 333 \\
\hline C. depressa (Hedw.) A. Jaeger & TR & $\mathrm{C}, \mathrm{EX}$ & $0-100$ & Neotropical; RR, RO, AC, AM, TO, PE, GO, MT, MS, PA e RJ & S \& V 266 \\
\hline C. martiana (Hornsch.) A. Jaeger & $\mathrm{TR}$ & $\mathrm{C}, \mathrm{R}$ & $0-1.000$ & Brasil; AP, BA, MT, MS, MG, RJ, PR, SC e RS & S \& V 90 \\
\hline C. merkelii (Hornsch.) A. Jaeger & $\mathrm{TR}$ & $\mathrm{C}, \mathrm{EX}$ & $0-800$ & Neotropical; RR, AC, PA, TO, AP, MG, RJ, SP e SC & S \& V 207 \\
\hline C. rufescens (Mitt.) A. Jaeger & TR & $\mathrm{C}, \mathrm{EX}$ & $0-200$ & Neotropical; PE, GO e RJ & S \& C 319 \\
\hline Crossomitrium patrisiae (Brid.) Müll. Hal. & $\mathrm{TR}$ & $\mathrm{C}, \mathrm{EF}$ & $0-800$ & $\begin{array}{l}\text { Neotropical; RR, RO, AC, AM, PA, TO, CE, PE, BA, GO, MT, RJ, } \\
\text { SP e SC }\end{array}$ & S \& V 160 \\
\hline Cyclodictyon albicans (Hedw.) O. Kuntze & $\mathrm{TR}$ & $\mathrm{EX}, \mathrm{R}$ & $800-2.200$ & Neotropical; AP, CE, MT, MS, MG, RJ, SP, PR, e RS & S \& V248 \\
\hline C. leucomitrium (Müll. Hal.) Broth. & $\mathrm{TR}$ & $\mathrm{C}$ & $0-1.000$ & Brasil; RJ, PR, SC e RS & S \& V 81 \\
\hline Lepidopilidium brevisetum (Hampe) Broth. & $\mathrm{TR}$ & $\mathrm{C}$ & $0-1.200$ & Brasil; MG, ES, RJ, SP, SC e RS & S \& V 87 \\
\hline L. caudicaule (Müll. Hal.) Broth. & $\mathrm{TR}$ & $\mathrm{C}$ & $1.200-1.700$ & Brasil; RJ e SC & S \& V 163 \\
\hline L. plebejum (Müll. Hal.) Sehnem & TR & $\mathrm{C}$ & $0-1.400$ & América do Sul; RJ, SC e RS & S \& V 71 \\
\hline Lepidopilum muelleri (Hampe) Spruce & $\mathrm{TR}$ & $\mathrm{C}, \mathrm{EX}$ & $800-1.300$ & México, Ilhas Coco e Brasil; MG e RJ & S \& V 242 \\
\hline L. scabrisetum (Schwägr.) Steere & $\mathrm{TR}$ & $\mathrm{C}, \mathrm{EX}$ & $0-1.700$ & Neotropical; RR, AP, AC, AM, PA, PE, BA, GO, MG, RJ, SP, SC e RS. & S \& V 231 \\
\hline L. subsubulatum Geh. \& Hampe & $\mathrm{TR}$ & $\mathrm{C}$ & $0-2.000$ & Brasil; AP, MG, RJ, SP, SC e RS & S \& C 380 p.p. \\
\hline Pilotrichum evanescens (Müll. Hal.) Müll. Hal. & $\mathrm{DN}$ & $\mathrm{C}$ & $0-1.200$ & Neotropical; RR, AP, AC, AM, PA, PE, BA, GO, MG, RJ, SP, PR e SC. & $\mathrm{S} \& \mathrm{C} 381$ \\
\hline Thamniopsis incurva (Hornsch.) W.R. Buck & TR & $\mathrm{R}, \mathrm{T}$ & $0-1.400$ & $\begin{array}{l}\text { Neotropical; AP, AM, PA, PB, PE, BA, GO, MT, MG, ES, RJ, SP, PR, } \\
\text { SC e RS }\end{array}$ & S \& V 185 \\
\hline T. stenodictyon (Sehnem) Oliveira-e-Silva \& Yano & $\mathrm{TR}$ & EX & $200-1.200$ & Brasil; RJ, SP, SC e RS & $\begin{array}{l}\mathrm{S} \& \mathrm{~V} 93 \\
\text { continua }\end{array}$ \\
\hline
\end{tabular}




\begin{tabular}{|c|c|c|c|c|c|}
\hline Espécies & FV & $\mathrm{TS}$ & Altitude $\mathrm{Br}(\mathrm{m})$ & Distribuição geográfica no mundo e no Brasil & Voucher \\
\hline \multicolumn{6}{|l|}{ POTTIACEAE (1/1) } \\
\hline Hyophilla involuta (Hook.) A. Jaeger & $\mathrm{TF}$ & $\mathrm{A}, \mathrm{R}$ & $0-700$ & $\begin{array}{l}\text { Ampla; RR, RO, AP, AM, PA, PI, CE, PB, PE, BA, GO, MS, MG, ES, RJ, } \\
\text { SP, PR e RS }\end{array}$ & S \& C 113 \\
\hline \multicolumn{6}{|l|}{ PTEROBRYACEAE (1/1) } \\
\hline $\begin{array}{l}\text { Pterobryon densum (Schwagr.) Hornsch. } \\
\text { RACOPILACEAE (1/1) }\end{array}$ & $\mathrm{DN}$ & $\mathrm{C}$ & $200-1.200$ & Neotropical; MG, RJ, SP, PR, SC e RS. & S \& C 249 \\
\hline Racopilum tomentosum (Hedw.) Brid. & $\mathrm{TP}$ & $\mathrm{R}, \mathrm{T}$ & $0-2.700$ & $\begin{array}{l}\text { Afro-americana; RR, RO, AP, AC, AM, PA, TO, CE, PE, BA, GO, MT, } \\
\text { MS, MG, ES, RJ, SP, PR, SC e RS }\end{array}$ & S \& C 206 \\
\hline \multicolumn{6}{|l|}{ RHYZOGONIACEAE $(1 / 1)$} \\
\hline $\begin{array}{l}\text { Pirrhobryum spiniforme (Hedw.) Mitt. } \\
\text { SEMATOPHYLLACEAE }(2 / 3)\end{array}$ & $\mathrm{TF}$ & $\mathrm{C}, \mathrm{R}$ & $0-2.200$ & Ampla; RO, AP, AC, PE, BA, GO, MT, MG, RJ, SP, PR, SC e RS & $\mathrm{S} \& \mathrm{C} 238$ \\
\hline Sematophyllum galipense (Müll. Hal.) Mitt. & TR & $\mathrm{R}$ & $0-800$ & $\begin{array}{l}\text { Neotropical; RR, RO, AP, AC, PA, TO, CE, PE, BA, GO, MT, MS, MG, } \\
\text { ES, RJ, SP, PR, SC e RS }\end{array}$ & S \& C 179 \\
\hline S. subpinnatum (Brid.) E. Britton & TRA & $\mathrm{C}, \mathrm{EF}, \mathrm{R}$ & $0-1.500$ & $\begin{array}{l}\text { Ampla; RR, RO, AP, AC, AM, PA, TO, CE, PB, PE, BA, GO, MT, MS, } \\
\text { MG, ES, RJ, SP, PR, SC e RS }\end{array}$ & S \& C 199 \\
\hline S. subsimplex (Hedw.) Mitt. & $\mathrm{TR}$ & EX & $0-2.000$ & $\begin{array}{l}\text { Pantropical; RR, RO, AP, AC, AM, PA, TO, MA, PI, CE, PB, PE, SE, BA, } \\
\text { GO, MT, MS, MG, RJ, SP, PR, SC e RS }\end{array}$ & S \& C 343 \\
\hline Taxithelium planum (Brid.) Mitt. & $\mathrm{TR}$ & $\mathrm{C}$ & $0-1.000$ & $\begin{array}{l}\text { Ampla; RR, RO, AP, AC, AM, PA, TO, PE, AL, BA, GO, MT, MS, MG, } \\
\text { RJ, SP, PR e SC }\end{array}$ & S \& C 318 \\
\hline \multicolumn{6}{|l|}{ STEREOPHYLLACEAE (1/1) } \\
\hline Pilosium chlorophyllum (Hornsch.) Müll. Hal. & $\mathrm{TP}$ & $\mathrm{R}$ & $0-800$ & $\begin{array}{l}\text { Neotropical (alcançando o sudeste dos EUA); RR, RO, AP, C, AM, PA, } \\
\text { TO, CE, PB, AL, BA, GO MT, MS, MG, ES, RJ e SP }\end{array}$ & S \& C 300 \\
\hline \multicolumn{6}{|l|}{ THUIDIACEAE (1/1) } \\
\hline $\begin{array}{l}\text { Cyrto-hypnum schistocalyx (Müll. Hal.) } \\
\text { W.R. Buck \& H.A. Crum }\end{array}$ & $\mathrm{TR}$ & $\mathrm{R}$ & $0-1.000$ & $\begin{array}{l}\text { Neotropical (alcançando o sudeste dos EUA); RO, AC, AM, PA, TO, MA, } \\
\text { PE, GO, MT, MS e RJ }\end{array}$ & S \& C 129 \\
\hline
\end{tabular}


Segundo Costa \& Lima (2005), a maior diversidade de espécies de Calymperes (Calymperaceae) e Callicostella (Pilotrichaceae) no Estado do Rio de Janeiro, ocorre na floresta submontana. Na área, esses gêneros estão bem representados, o primeiro com três espécies e o segundo com cinco, corroborando as observações das autoras.

A elevada riqueza de espécies observada na família Aneuraceae, representada por nove das 11 espécies conhecidas para o Estado (81\%), possivelmente está relacionada ao alto grau de umidade e disponibilidade de microambientes favoráveis ao desenvolvimento desses táxons na área, como barrancos úmidos ao longo das trilhas e riachos, e rochas nas margens de córregos e no interior da mata.

Formas de vida - Foram caracterizados sete tipos de formas de vida: coxim, dendróide, pendente, talosa, tapete, trama e tufo (Tab. 1); predominando trama, com 50 espécies; seguida de tufo, com 22 espécies; talosa, com 21 espécies; pendente e tapete, com 18 espécies cada (Fig. 1).

Segundo Mägdefrau (1982), as formas de vida de briófitas estão diretamente relacionadas à disponibilidade de luz e umidade no ambiente. Na RPPN El Nagual, as espécies do tipo trama ocorrem, principalmente, como corticícolas ou rupícolas nas áreas abertas, como capoeira, borda da mata e margem do Rio das Pedras Negras. Essa forma de vida é capaz de armazenar consideráveis quantidades de água da chuva pela ação da capilaridade, e em geral é observada em áreas com alta intensidade luminosa e elevada umidade do ar (Mägdefrau 1982).

Os tufos, que na RPPN são formados exclusivamente por espécies de musgos (totalizando $33 \%$ do grupo), ocorrem principalmente como rupícolas nas formações de capoeira e na base de troncos de

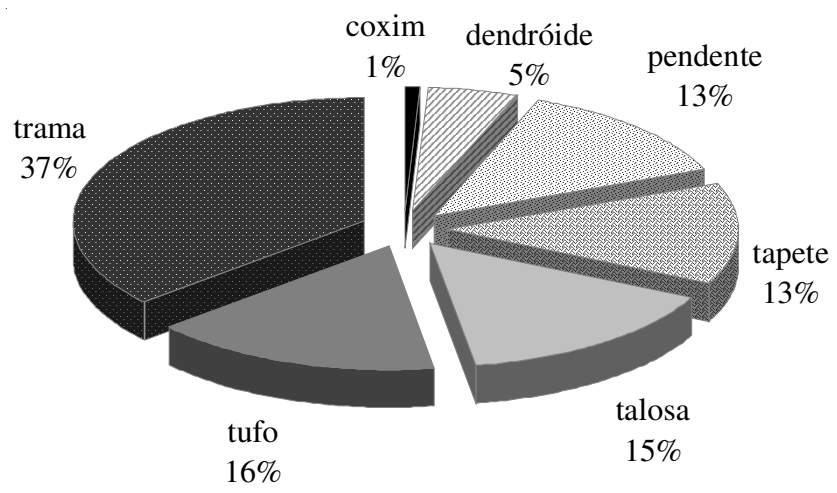

Figura 1. Formas de vida das espécies de briófitas da RPPN El Nagual, Magé, RJ, Brasil. árvores, rochas e barrancos nas trilhas no interior da floresta. Todas as espécies de Bryaceae, Calymperaceae, Daltoniaceae e Dicranaceae encontradas na área, formam tufos. Em geral, espécies com esse tipo de forma de vida suportam condições de maior estresse hídrico, devido ao fato dos gametófitos estarem aglomerados podendo armazenar água e conduzi-la por capilaridade (Mägdefrau 1982).

Apesar de trama e tufo terem predominado (53\% dos táxons), as formas de vida talosa e pendente também se destacam pelo número de espécies, revelando por um lado, que a área apresenta disponibilidade de substratos típicos para talosas, como rochas e barrancos à margem de córregos e rios, e por outro, elevada umidade atmosférica, relacionada com a presença de um grande número de pendentes (18 táxons), que são características de florestas tropicais úmidas, crescendo em locais sombreados e com umidade do ar alta.

Substrato - As espécies na área estudada colonizam seis tipos de substrato (Tab. 1 e Fig. 2), predominando corticícolas, seguidas de rupícolas, epíxilas, terrícolas e epífilas. Dentre as 137 espécies, 103 (75\%) ocorreram em um único tipo de substrato, predominando as corticícolas. Três espécies, Hyophyla involuta, Philonotis uncinata e Sematophyllum subpinnatum, ocorreram em substrato artificial, paredão de cimento da represa do Rio das Pedras Negras. Essas espécies são freqüentemente encontradas nesse tipo de substrato em áreas urbanas (Molinaro \& Costa 2001).

O elevado número de espécies corticícolas (52\% da brioflora) era esperado, visto que em florestas tropicais, a disponibilidade desse tipo de substrato é alta. Assim como o baixo número de espécies terrícolas (12\% da brioflora), cuja existência de uma camada de serrapilheira no solo dessas florestas, dificulta o crescimento das briófitas.

As rupícolas também estão bem representadas, com $42 \%$ da brioflora, isso devido a grande disponibilidade de superfícies rochosas nos rios, córregos e nascentes da área. Plagiochila patula, uma espécie típica de rochas úmidas (Gradstein \& Costa 2003), foi encontrada somente sobre rochas no córrego do interior da floresta. As hepáticas talosas, Aneura pinguis, Dumortiera hirsuta, Marchantia chenopoda, Monoclea gottschei e Symphyogyna aspera, e os musgos, Cyrto-hypnum schistocalyx e Plagiomnium rhynchophorum, ocorreram somente sobre as rochas do Rio das Pedras Negras, enquanto 


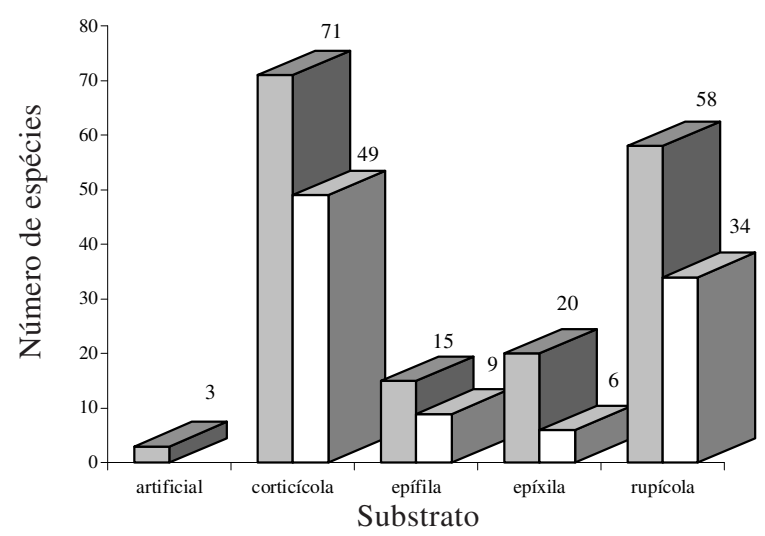

Figura 2. Substratos colonizados pelas espécies de briófitas na RPPN El Nagual, Magé, RJ, Brasil. ( $\square=$ Tipo de substrato $\square=$ Exclusivas por substrato).

que o antócero, Megaceros vincentianus, ocorreu sobre rocha de um córrego na margem da floresta.

O número considerável de epífilas observado (15 espécies - 11\%) indica o bom estado de conservação da área, visto que essa comunidade é a mais vulnerável, desaparecendo rapidamente após distúrbios ambientais, como abertura de clareiras, desmatamento, fragmentação da mata, etc. (Pócs 1996; Gradstein et al. 2001; Zartman 2003). Segundo Gradstein \& Pócs (1989), briófitas epífilas são características de florestas tropicais úmidas, e a presença de uma comunidade rica está relacionada à alta umidade atmosférica. $\mathrm{Na}$ área de estudo foram encontradas duas espécies consideradas epífilas obrigatórias, Cololejeunea subcardiocarpa e Drepanolejeunea biocellata, além de duas epífilas facultativas comuns nas florestas neotropicais, Crossomitrium patrisiae e Leptolejeunea elliptica (Gradstein \& Costa 2003; Pócs 1996).

Gradstein et al. (2001) comentam que as espécies de briófitas epífitas (corticícolas, epífilas e epíxilas), constituem a maior parte da brioflora das florestas úmidas e não se distribuem aleatoriamente dentro da floresta. Segundo esses autores, muitas espécies ocorrem exclusivamente em locais úmidos e sombrios do interior da mata (epífitas de sombra), enquanto que outras ocorrem em locais mais secos e expostos à alta intensidade luminosa, como o dossel da mata (epífitas de sol), e ainda aquelas que ocorrem nos dois ambientes (generalistas). $\mathrm{Na}$ reserva foram encontradas oito espécies consideradas epífitas de sombra: Archilejeunea parviflora, Bryopteris filicina, Plagiochila corrugata, P. patula, P. raddiana, P. subplana, Phyllogonium viride e Taxithelium planum; cinco epífitas de sol: Diplasiolejeunea alata e todas as espécies de Frullania; e nove generalistas: Ceratolejeunea cornuta, C. cubensis, Drepanolejeunea biocellata, Lejeunea laetevirens, Metzgeria ciliata, Octoblepharum albidum, Plagiochila martiana, Radula nudicaulis e Symbiezidium barbiflorum.

Segundo Gradstein (1992), as epífitas de sombra são as primeiras que desaparecem em caso de distúrbios na floresta, podendo ser utilizadas como bioindicadoras de áreas primárias ou em estágios avançados de regeneração. $\mathrm{O}$ número de epífitas de sombra encontrado na reserva está relacionado ao seu bom estado de conservação, com áreas de florestas primárias e áreas de florestas em estágio de regeneração avançado.

Padrões de distribuição - Foram caracterizados cinco padrões de distribuição (Tab. 1), predominando o Neotropical com 64 espécies (47\%), seguido do Pantropical com 18 espécies (13\%), Amplo com 14 espécies (10\%), Restrito ao Brasil com 12 espécies (9\%), e Afro-americano com 10 espécies (7\%). Logo, $60 \%$ das espécies encontradas apresentam uma distribuição relativamente ampla nos trópicos, resultado similar ao encontrado em outros trabalhos realizados em florestas tropicais (Costa \& Silva 2003; Costa \& Lima 2005; Gradstein et al. 1989; Gradstein \& SalazarAllen 1992; Ilkiu-Borges et al. 2004; Valente \& Pôrto 2006 etc.).

Amblystegium cf. serpens, Diplasiolejeunea cavifolia, Drepanolejeunea biocellata, Megaceros vicentianus e Taxilejeunea obtusangula são novos registros para o Estado do Rio de Janeiro, demonstrando por um lado, a importância do remanescente florestal estudado, e por outro, que a brioflora do Estado ainda necessita de estudos florísticos.

Drepanolejeunea biocellata é uma espécie Neotropical, epífila, conhecida para o Brasil por apenas coleções antigas do Estado de São Paulo, entre 0$900 \mathrm{~m}$. Foi encontrada na capoeira, sobre folha de arbusto, sendo essa a coleção mais recente realizada para o país após 50 anos.

Megaceros vincentianus, uma espécie com distribuição Neotropical, e que no Brasil era conhecida apenas por duas coleções, uma realizada a mais de 100 anos para Pernambuco e outra a 20 anos para São Paulo, entre 500-1.500 m (Gradstein \& Costa 2003), foi encontrada na área ocorrendo a 210 m, sobre rocha úmida. Essa espécie vem sendo encontrada, ultimamente, em diferentes localidades do Estado do Rio de Janeiro (PARNAs Itatiaia e Tijuca, PE Desengano), entre 200-1.100 m. Assim sendo, a atual 
distribuição (PE, RJ e SP) possivelmente não reflete a distribuição real do táxon, visto que, a ausência do esporófito que apresenta esporos verdes e elatérios com uma espiral de espessamento, dificulta sua identificação.

Riccardia amazonica, $R$. emarginata, Plagiochila raddiana, Vesicularia vesicularis var. portoricensis e Callicostella apophysata, anteriormente citadas sem localidade para o Estado do Rio de Janeiro (Costa et al. 2005b), foram encontradas na área de estudo, sendo agora conhecidas para o município de Magé.

Variação altitudinal - $\mathrm{O}$ limite altitudinal de ocorrência no Brasil de alguns táxons encontrados na área de estudo foi ampliado, visto que, diversas espécies foram encontradas a ca. $200 \mathrm{~m}$ na RPPN: Bazzania longistipula (400-2.400 m), Cyclodictyon albicans (800-2.200 m), Diplasiolejeunea alata (700-1500 m), Lejeunea $\mathrm{cf}$. cristulata (900-1.500 m), Lepidopilidium caudicaule (1.200-1.700 m), Lepidopilum muelleri (800-1.300 m), Lophocolea mandonii (1.000-2.500 m), Megaceros vincentianus (500-1.500 m), Porotrichum longirostre (800-2.700 m), Radula nudicaulis (800-2.700 m), e $R$. recubans (800-1.000 m).

Status de conservação - Com base nos dados de Costa et al. (2005b) para o Estado do Rio de Janeiro, foram encontradas na área de estudo quatro espécies caracterizadas como vulneráveis. Lophocolea mandonii, conhecida para Bolívia e sudeste do Brasil (MG e RJ), no Estado ocorre somente na Serra de Itatiaia, acima de $1.300 \mathrm{~m}$ (Fulford 1976), foi encontrada na reserva a $270 \mathrm{~m}$. Aphanolejeunea paucifolia, conhecida para Argentina, Paraguai e Brasil (MG, ES, RJ e SP), citada no Estado somente para o arboreto do Jardim Botânico do Rio de Janeiro, foi encontrada na reserva ocorrendo sobre folha, na margem de um córrego no interior da floresta. Plagiochila patula, espécie neotropical, ocorrendo no Brasil nos Estados do AC, BA, MG, RJ e SP, e no Rio de Janeiro nos municípios de Itatiaia e Nova Friburgo (ca. $900 \mathrm{~m}$ ), foi encontrada na reserva sobre rocha, na margem do Rio das Pedras Negras, a 190 m. Lepidopilidium caudicaule, espécie endêmica do Brasil (RJ e SC), conhecida no Estado somente para a Serra dos Órgãos, entre 1.200-1.700 m, foi encontrada na reserva a $200 \mathrm{~m}$, sobre raiz de uma epífita na trilha.

Os resultados aqui apresentados demonstram que a brioflora da RPPN El Nagual é rica, visto que numa área pequena foram encontradas $13 \%$ da brioflora do Estado e 54\% daquela conhecida para a Floresta
Atlântica submontana do Estado do Rio de Janeiro. Essa riqueza está possivelmente relacionada ao bom estado de conservação da área, ao fato da RPPN El Nagual pertencer a Serra dos Órgãos e aos diferentes ambientes (córregos, rios, florestas e capoeiras), que fornecem uma diversidade de microhabitats e fontes alternativas para a manutenção da umidade do ar, fundamentais ao desenvolvimento das briófitas. Além disso, demonstram o alto valor de conservação dessa área de floresta submontana, visto que nela foram encontradas cinco novas ocorrências de briófitas para o Estado, além de espécies consideradas vulneráveis no Rio de Janeiro. Evidencia-se assim, a importância de RPPNs no fortalecimento do sistema de proteção da natureza, na conservação da diversidade da brioflora e na proteção de remanescentes de Mata Atlântica no Estado do Rio de Janeiro. Por outro lado, os resultados demonstram a necessidade de levantamentos brioflorísticos em partes do Estado.

\section{Agradecimentos}

Ao Instituto de Pesquisas Jardim Botânico de Rio de Janeiro e à Fundação $\mathrm{O}$ Boticário de Proteção à Natureza, pelo financiamento da pesquisa (projeto 0709_20061); à Mariana de Calloch e Horst B. Calloch, proprietários da RPPN El Nagual, por permitirem a realização deste estudo.

\section{Referências bibliográficas}

Bartram, E.B. 1954. Musci. In: C.T. Rizzini (ed.). Flora Organensis. Lista dos Cormophyta da Serra dos Órgãos. Arquivos do Jardim Botânico do Rio de Janeiro 13: 234-242.

Brotherus, V.F. 1924. Ergebnisse der botanischen Expedition der Kaiserlichen Akademie der Wissenschaften nach Südbrasilien 1901, heralsgegeben von Prof. Dr. V. Schiffner. Denkschr. Akademie der Wissenschaften in Wien 83: 251-358.

Buck, W.R. 1998. Pleurocarpous Mosses of the West Indies. Memoirs of The New York Botanical Garden 1: $1-401$.

Buck, W.R. \& Goffinet, B. 2000. Pp. 71-123. Morphology and classification of mosses. In: A.J. Shaw \& B. Goffinet (eds.). Bryophyte Biology. England, Cambridge University Press.

Costa, D.P. 1988. Leucobryaceae do Parque Nacional da Tijuca no Estado do Rio de Janeiro (Brasil). Rodriguésia 64/66(41/40): 41-48.

Costa, D.P. 1992. Hepáticas do Pico da Caledônea. Nova Friburgo, Rio de Janeiro, Brasil. Acta Botanica Brasilica 6: 3-39. 
Costa, D.P. 1994. Musgos do Pico da Caledônea, município de Nova Friburgo, Estado do Rio de Janeiro, Brasil. Acta Botanica Brasilica 8: 141-191.

Costa, D.P. 1997. Bryopyta e Hepatophyta. Pp. 37-43. In: M.C.M. Marques (ed.). Mapeamento da cobertura vegetal e listagem das espécies ocorrentes na APA Cairuçu, Parati, RJ. Série Estudos e Contribuições n. 13, Rio de Janeiro.

Costa, D.P. 1999. Epiphytic bryophyte diversity in primary and secondary lowland rainforests in southeastern Brazil. Bryologist 102: 320-326.

Costa, D.P. 2007. Metzgeriaceae. Flora Neotropica. Monograph 102. New York, The New York Botanical Garden.

Costa, D.P. \& Yano, O. 1988. Hepáticas talosas do Parque Nacional da Tijuca, Rio de Janeiro, Brasil. Acta Botanica Brasilica 1: 73-82.

Costa, D.P. \& Yano, O. 1995. Musgos do município de Nova Friburgo, Rio de Janeiro, Brasil. Arquivos do Jardim Botânico do Rio de Janeiro 33: 99-118.

Costa, D.P. \& Yano, O. 1998. Briófitas da restinga de Macaé, Rio de Janeiro, Brasil. Hoehnea 25: 99-119.

Costa, D.P. \& Salamene, S. 2003. Base de Dados do Projeto Paisagem e Flora da Reserva Biológica do Tinguá: subsídios ao monitoramento da vegetação. Instituto de Pesquisas Jardim Botânico do Rio de Janeiro.

Costa, D.P. \& Silva, A.G. 2003. Briófitas da Reserva Natural da Vale do Rio do Doce, Linhares, Espírito Santo, Brasil. Boletim do Museu de Biologia Mello Leitão 16: 21-38.

Costa, D.P. \& Lima, F.M. 2005. Moss diversity in the tropical rainforests of Rio de Janeiro, Southeastern Brazil. Revista Brasileira de Botânica 28: 671-685.

Costa, D.P.; Imbassahy, C.A.A. \& Silva, V.P.A.V. 2005a. Checklist and distribution of mosses, liverworts and hornworts of Rio de Janeiro state, Brazil. The Journal Hattori Botanical Laboratory 98: 259-298.

Costa, D.P.; Imbassahy, C.A.A. \& Silva, V.P.A.V. 2005 b. Diversidade e importância das espécies de briófitas na conservação dos ecossistemas do Estado do Rio de Janeiro. Rodriguésia 56: 13-49.

Costa, D.P.; Imbassahy, C.A.A.; Almeida, J.S.S.; Santos, N.D.; Vaz-Imbassahy, T.F. 2006. Diversidade das briófitas nas restingas do Estado do Rio de Janeiro. Boletim do Instituto de Botânica 18: 131-140.

Crandall-Stotler, B. \& Stotler, R.E. 2000. Pp. 21-70. Morphology and classification of the Marchantiophyta. In: A.J. Shaw \& B. Goffinet (eds.). Bryophyte Biology. England, Cambridge University Press.

Crum, H. \& Anderson, L.E. 1981. Mosses of eastern North America. 2 v. New York, Columbia University Press.

Dauphin, G.L. 2003. Ceratolejeunea. Flora Neotropica. Monograph 90. New York, The New York Botanical Garden.

Dusén, P. 1903. Sur la flore de la Serra do Itatiaya au Brésil. Arquivos do Museu Nacional do Rio de Janeiro 13: 1-119.

Frahm, J.-P. 1991. Dicranaceae: Campylopodioideae, Paraleucobryoideae. Flora Neotropica. Monograph 54. New York, The New York Botanical Garden.
Fulford, M. 1976. Manual of Hepaticae of Latin America. Part IV. Memories of The New York Botanical Garden 11: 393-535.

Fundação SOS Mata Atlântica. 2002. Atlas da evolução dos Remanescentes Florestais e Ecossistemas associados da Mata Atlântica no período 1995-2000. São Paulo, Fundação S.O.S. Mata Atlântica/INPE.

Gradstein, S.R. 1992. The vanishing tropical rain forest as an environment for bryophytes and lichens. Pp. 234-258. In: J.W. Bates \& A.W. Farmer (eds.). Bryophytes and Lichens in a Changing Environment. Oxford.

Gradstein, S.R. 1995. Bryophyte diversity of the tropical rainforest. Archives des sciences [Société de physique et d'histoire naturelle de Genève] 48: 91-96.

Gradstein, S.R. \& Pócs, T. 1989. Bryophytes. Pp. 311-325. In: H. Lieth \& M.J.A. Werger (eds.). Tropical rain forest ecosystems. Amsterdan, Elsevier.

Gradstein, S.R. \& Salazar-Allen, N. 1992. Bryophyte diversity along an altitudinal gradient in Dárien National Park, Panama. Tropical Bryology 5: 61-71.

Gradstein, S.R.; van Rennen, G.B.A. \& Griffin III, D. 1989. Species richness and origin of the bryophyte flora of the Colombian Andes. Acta Botanica Neerlandica 38: 439-448.

Gradstein, S.R.; Churchill, S.P. \& Salazar-Allen, N. 2001. Guide to the Bryophytes of Tropical America. Memoirs of The New York Botanical Garden 86: 1-577.

Gradstein, S.R. \& Costa, D.P. 2003. The Hepaticae and Anthocerotae of Brazil. Memoirs of The New York Botanical Garden 87: 1-301.

Hedenäs, L. 2003. Amblystegiaceae. Flora Neotropica. Monograph 89. New York, The New York Botanical Garden.

Heinrichs, J.; Gradstein, S.R. \& Grolle, R. 1988. A revision of the neotropical species of Plagiochila (Dumort.) Dumort. (Hepaticae) described by Olof Swartz. Journal of the Hattori Botanical Laboratory 85: 1-32.

Heinrichs, J.; Anton, H.; Gradstein, S.R. \& Mues, R. 2000. Systematics of Plagiochila sect. Glaucescentes Carl (Hepaticae) from Tropical America: a morphological and chemotaxonomical approach. Plant Systematic and Evolution 220: 115-138.

Hell, K.G. 1969. Briófitas talosas dos arredores da cidade de São Paulo, Brasil. Universidade de São Paulo. Boletim da Faculdade de Filosofia, Ciências e Letras 25: 1-190.

Herzog, T. 1925. Neue Bryophyten aus Brasilien. Repertorium specierum novarum regni vegetabilis 21 : 22-38.

Hornschuch, C.F. 1840. Musci. Pp. 1-100. In: C.F.P. Martius (ed.). Flora Brasiliensis enumeratio plantarum in Brasilia hactenus detectarum quas suis aliorumque botanicorum studiis descriptas et methodo naturali digestas partim icone illustratas. v. 1(2). Monachii.

Ilkiu-Borges, A.L.; Tavares, A.C.C. \& Lisboa, R.C.L. 2004. Briófitas da Ilha de Germoplasma, reservatório de Tucuruí, Pará, Brasil. Acta Botanica Brasilica 18: 689-692.

Mägdefrau, K. 1982. Life forms of bryophytes. Pp. 45-58. In: A.J.E. Smith (ed.). Bryophyte Ecology. New York. 
Ministério do Meio Ambiente \& O Instituto Ambiental. 2005. Plano de Manejo e Zoneamento da Reserva Particular do Patrimônio Natural El Nagual. Convênio 24/2003 (inédito).

Molinaro, L.C. \& Costa, D.P. 2001. Briófitas do arboreto do Jardim Botânico do Rio de Janeiro. Rodriguésia 52: 107-124.

Müller, C. 1898. Bryologia Serrae Itatiaiae. Bulletin of the Herbier Boissier 6: 18-48.

Müller, C. 1900. Symbolae ad bryologiam Brasiliae et regionum vicinarum. Hedwigia 39: 235-289.

Müller, C. 1901. Symbolae ad bryologiam Brasiliae et regionum vicinarum. Hedwigia 40: 55-99.

Nees ab Esenbeck, C.G. 1833. Hepaticae. Pp. 293-390. In: C.F.P. Martius (ed.). Flora Brasiliensis. Stuttgartiae et Turbingae. J. G. Cottal ed. 1.

Ochi, H. 1980. A revision of the Neotropical Bryoideae, Musci (First Part). The Journal of the Faculty of Education, Tottori University, Natural Science 29: 49-154.

Ochi, H. 1981. A revision of the Neotropical Bryoideae, Musci (Second Part). The Journal of the Faculty of Education, Tottori University, Natural Science 30: 21-55.

Oliveira-e-Silva, M.I.M.N \& Yano, O. 2000a. Anthocerotophyta e Hepatophyta de Mangaratiba e Angra dos Reis, Rio de Janeiro, Brasil. Boletim do Instituto de Botânica 13: 1-102.

Oliveira-e-Silva, M.I.M.N \& Yano, O. 2000b. Musgos de Mangaratiba e Angra dos Reis, Rio de Janeiro, Brasil. Boletim do Instituto de Botânica 14: 1-137.

Pócs, T. 1982. Tropical forest bryophytes. Pp. 59-104. In: A.J.E. Smith (ed.). Bryophyte Ecology. London.

Pócs, T. 1996. Epiphyllous liverwort diversity at worldwide level and its threat and conservation. Anales del Instituto Nacionale Autómono de Biologia, Serie Botánica 67: 109-127.

Raddi, G. 1822. Crittogame brasiliane racclote e descritte (preprint) (Também publicado em: 1823. Memorie di matematica e di fisica della società italiana delle scienze residente in Modena 19: 27-57).

Rambaldi, D.M.; Magnani, A.; Ilha, A.; Lardosa, E.; Figueiredo, P.; Oliveira, R.F. 2003. A Reserva da Biosfera da Mata Atlântica no Estado do Rio de Janeiro. Série Estados e Regiões da RBMA. Caderno da Reserva da Biosfera da Mata Atlântica. Rio de Janeiro, CNRBMA.

Reese, W.D. 1993. Calymperaceae. Flora Neotropica. Monograph 58. New York, The New York Botanical Garden.

Reiner-Drehwald, M.E. 2000. Las Lejeuneaceae (Hepaticae) de Missiones, Argentina VI. Lejeunea y Taxilejeunea. Tropical Bryology 19: 81-131.

Reiner-Drehwald, M.E. \& Goda, A. 2000. Revision of the genus Crossotolejeunea (Lejeuneaceae, Hepaticae). The Journal Hattori Botanical Laboratory 89: 1-54.
Richards, P.W. 1954. Notes on the bryophytes communites of low-land tropical rain forests, with special reference to Moraballi Creek, British Guyana. Vegetatio 5-6: 319-328.

Richards, P.W. 1984. The Ecology of Tropical Forest bryophytes. Pp. 233-1270. In: R.M. Schuster (ed.). New Manual of Bryology. v. 1. Japan, Hattori Botanical Laboratory.

Robbins, R.G. 1952. Bryophyte ecology of a dune area in New Zealand. Vegetatio, Acta Geobotanica 4: 1-31.

Sharp, A.J.; Crum, H.A. \& Eckel, P.M. 1994. The Moss Flora of Mexico. Memoirs of the New York Botanical Garden 69: 1-1113.

Stephani, F. 1905-1909. Species Hepaticarum 3: 1-693.

Stephani, F. 1909-1912. Species Hepaticarum 4: 1-824.

Stotler, R.E. \& Crandall-Stotler, B. 2005. A revised classification of the Anthocerotophyta and a checklist of the hornworts of North America, north of Mexico. Bryologist 108: 16-26.

Valente, E.B. \& Pôrto, K.C. 2006. Hepáticas (Marchantiophyta) de um fragmento de Mata Atlântica na Serra da Jibóia, município de Santa Teresinha, Bahia, Brasil. Acta Botanica Brasilica 20: 433-441.

Vaz-Imbassahy, T.F. \& Costa, D.P. 2007a. Os gêneros Brymela, Callicotella, Crossomitrium, Cyclodictyon, Hookeriopsis e Hypnella no Estado do Rio de Janeiro. Acta Botanica Brasilica 20: 955-973.

Vaz-Imbassahy, T.F. \& Costa, D.P. 2007b. Os gêneros Lepidopilidium, Lepidopilum, Pilotrichum e Thamniopsis no Estado do Rio de Janeiro. Acta Botanica Brasilica 20: 975-993.

Veloso, H.P.; Rangel Filho, A.L.R. \& Lima, J.C.A. 1991. Classificação da Vegetação Brasileira adaptada a um Sistema Universal. Rio de Janeiro, IBGE/CDDI. Departamento de Documentação e Biblioteca.

Yano, O. 1981. A checklist of Brazilian mosses. The Journal of the Hattori Botanical Laboratory 50: 279-456.

Yano, O. 1984a. Briófitas. Pp. 27-30. In: O. Fidalgo \& V.L.R. Bononi (eds.). Técnicas de coleta, preservação e herborização de material botânico. Série Documentos. São Paulo, Instituto de Botânica.

Yano, O. 1984b. Checklist of Brazilian liverworts and hornworts. The Journal of the Hattori Botanical Laboratory 56: 481-548.

Yano, O. 1989. An additional checklist of Brazilian bryophytes. The Journal of the Hattori Botanical Laboratory 66: 371-434.

Yano, O. 1995. A new additional annotated checklist of Brazilian bryophytes. The Journal of the Hattori Botanical Laboratory 78: 137-182.

Yano, O. 2006. Novas adições ao catálogo de briófitas brasileiras. Boletim do Instituto de Botânica 17: 1-142.

Zartman, C.E. 2003. Habitat fragmentation impacts on epiphyllous bryophyte communities in Central Amazonia. Ecology 84: 948-954. 